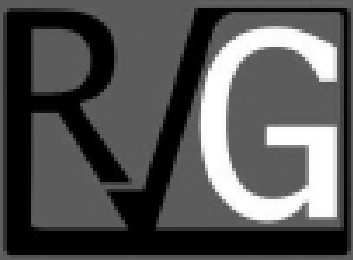

Año 24 No. 88

Octubre - Diciembre 2019

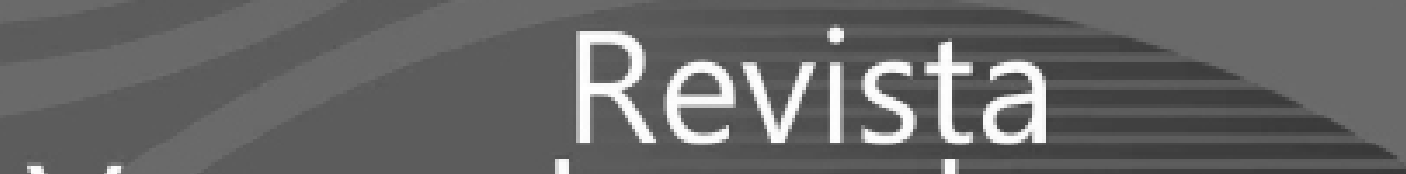

Venezolana de

verencla

UNIVERSIDAD DEL ZULIA (LUZ)

Facultad de Ciencias Económicas y Sociales

Centro de Estudios de la Empresa 


\title{
Proceso de consultoría organizacional: modelo conceptual
}

\author{
Margarita Guerrero Aguiar ${ }^{1}$ \\ Alberto Medina León ${ }^{2}$ \\ Dianelys Nogueira Rivera ${ }^{3}$ \\ Rafael Humberto Soler González ${ }^{4}$
}

\section{Resumen}

En el presente artículo caracteriza el proceso de consultoría organizacional con la finalidad de proponer un modelo para sistematizarlo. Se aplica la metodología clúster y el análisis de clasificación por conglomerados jerárquicos por casos, para obtener, desde la perspectiva teórica las variables más representativas existentes en los modelos y procedimientos precedentes, con la asistencia del software IBM Statistical Package for the Social Sciences Statistics (SPSS), versión 21. En su conformación se utilizan técnicas cualitativas como la tormenta de ideas, encuesta y el trabajo en grupo. Como principal resultado se presenta un modelo conceptual para desarrollar los servicios de consultoría organizacional que se sustenta en los elementos esenciales más repetitivos e invariantes de los 21 modelos y procedimientos analizados, que integra las etapas generalmente aceptadas y las mejores prácticas del equipo consultor. El modelo propuesto, logra uniformidad al sistematizar la forma de desarrollar los servicios de consultoría, que se articula con el merco legal y está orientado al cliente, quedando constituido por cinco fases: concertación del servicio, exploración, planificación, ejecución e informe gerencial, entrega y conformación del expediente.

Palabras clave: consultoría organizacional; modelo de consultoría; proceso de consultoría.

\footnotetext{
Recibido: 20-03-19 Aceptado: 25-07-19
}

\footnotetext{
Profesor Asistente. Universidad de Cienfuegos. Sociedad AUDITA S.A. Cimex, Cienfuegos. E-mail: margag@cimex.com.cu; mgaguiar@ucf.edu.cu.

Profesor Titular. Universidad de Matanzas. E-mail: alberto.medina@umcc.cu.

Profesor Titular. Universidad de Matanzas.E-mail: dianelys.nogueira@umcc.cu.

Profesor Principal. Universidad Metropolitana de Ecuador. Email: rsoler@umet.edu.ec
} 


\title{
Organizational consulting process: conceptual model
}

\begin{abstract}
This article characterizes the organizational consulting process with the purpose of proposing a model to systematize it. The cluster methodology and classification analysis by hierarchical clusters by cases are applied to obtain, from the theoretical perspective, the most representative variables existing in the preceding models and procedures, with the assistance of the IBM Statistical Package for the Social Sciences Statistics (SPSS) software ), version 21. Qualitative techniques such as brainstorming, survey and group work are used in its formation. As a main result, a conceptual model is presented to develop organizational consulting services that are based on the most repetitive and invariant essential elements of the 21 models and procedures analyzed, which integrates the generally accepted stages and best practices of the consulting team. The proposed model achieves uniformity in systematizing the way of developing consulting services, which is articulated with the legal merit and is oriented to the client, being constituted by five phases: service coordination, exploration, planning, execution and management report, delivery and conformation of the file.
\end{abstract}

Keywords: Organizational consulting; consulting model; consultancy process.

\section{Introducción}

La consultoría es un servicio de asesoría profesional independiente que ayuda a la empresa a: alcanzar los objetivos, mediante la solución de problemas; descubrir y evaluar oportunidades; mejorar el aprendizaje y lograr la puesta en práctica de los cambios (Kubr, 2002), como actividad es realizada por una o más personas, en una entidad, para contribuir a la solución de problemas o mejorar el desempeño (Mas, 2008).

"Es un servicio profesional, un proceso de retroalimentación donde el consultor aprende de los miembros de la organización y éstos a su vez, guiados por el consultor, pueden generar un estado de sinergias que permita activar el conocimiento acumulado en el personal" (Guerrero et al 2014:4). Durante el proceso, ocurre un intercambio de conocimientos, habilidades y aptitudes en el que ambos aprenden a penetrar en los problemas y a encontrar las soluciones viables para resolverlos (Guerrero, 2017:7).

La literatura sobre consultoría es amplia; sin embargo, cuando se trata del uso de modelos y procedimientos específicos sobre cómo llevar a cabo el proceso, la información disponible se reduce. De hecho, se plantea que "cada empresa consultora tiene un modelo de negocio propio y distinto de la competencia" (Mas, 2008:101). Adicionalmente, se considera que es un 
proceso que debe estar alineado con el marco legal regulatorio propio de cada país (Guerrero, 2018).

El concepto de consultoría evoluciona en el tiempo y, en la actualidad, se aprecian definiciones que ven al consultor involucrado tanto en el diseño de propuestas, como en la puesta en práctica de las acciones de mejora, con miras a capacitar y entrenar en el terreno, donde el consultor acompaña al cliente a través de la formación - acción. En referencia, la consultoría, vista desde la concepción innovadora es entendida como transferencia tecnológica al cliente.

De ahí, la necesidad de estudiarla como proceso organizacional que arroja resultados favorables en las organizaciones; su análisis crítico a nivel de los instrumentos metodológicos estudiados para proponer un modelo de consultoría, merece prestarle atención. Por ello, a partir de su estudio, se propone un modelo para sistematizar el desarrollo de los servicios de consultoría organizacional, que sirva de guía y contribuya a la formación de consultores mediante la transmisión del conocimiento, que se articule con el marco legal, como contribución a la satisfacción de las necesidades y expectativas de los clientes, al brindar servicios de mayor calidad que permitan aprovechar las mejoras prácticas existentes en el equipo consultor.

Para el desarrollo de esta investigación se parte de realizar los siguientes pasos: 1) Análisis y síntesis de los modelos y procedimientos de consultoría precedentes; 2) Detección de las variables más representativas utilizadas al efecto; 3) Trabajo grupal y análisis crítico con los consultores; 4) Propuesta de modelo de consultoría.

\section{Modelos y procedimientos de consultoría precedentes}

Para el diseño del modelo de consultoría organizacional se parte del estudio de 21 modelos, y procedimientos, en lo adelante instrumentos metodológicos, utilizados indistintamente en investigaciones precedentes, para lograr el desarrollo y mejoramiento organizacional; se expone su factibilidad de uso en instituciones educacionales, empresas y otros sectores (cuadro 1).

\section{Cuadro 1.}

Instrumentos metodológicos empleados en la consultoría

\begin{tabular}{|c|c|c|c|}
\hline $\mathrm{N}^{\circ}$ & $\begin{array}{l}\text { Instrumen- } \\
\text { tos metodo- } \\
\text { lógicos }\end{array}$ & Aportes fundamentales & Variables presentes \\
\hline 1 & $\begin{array}{c}\text { Schein } \\
(1988)\end{array}$ & $\begin{array}{l}\text { Propone tres tipos de consultoría (médico } \\
\text { paciente, experto y consultoría de proce- } \\
\text { sos), enfatiza que el último es idóneo para } \\
\text { una ayuda eficaz, donde el cliente participa } \\
\text { directamente en el proceso para aprender } \\
\text { a identificar sus problemas y encausarlos }\end{array}$ & $\begin{array}{l}\text { Proceso, retroalimentación, me- } \\
\text { jora, cambio, capacitación, cali- } \\
\text { dad del servicio, mejora }\end{array}$ \\
\hline
\end{tabular}




\section{Cont... Cuadro 1.}

$2 \quad \begin{gathered}\text { Herrero } \\ (1996)\end{gathered}$

3

\section{González}

(2000)

$4 \quad \operatorname{Kubr}(2002)$

6 Leiva (2007)

$8 \quad$ Mas (2008)
Analiza los aportes de las ciencias humanas a la consultoría de procesos, se abordan diferentes modelos de intervención para lograr el mejoramiento de la organización

Presenta un modelo de consultoría organizacional-gerencial con enfoque sistémico y de procesos para facilitar el cambio de la empresa cliente

Expone un modelo de consultoría que contiene cinco etapas: Iniciación, diagnóstico, planificación de acciones, aplicación y terminación y se explican cada una de ellas

Plantea que la implantación de la norma no ofrece rentabilidad económica, el beneficio se traduce en el aumento del conocimiento de la norma y sobre la gestión de la calidad en general, analiza modelos estándares de calidad

Diseña un modelo de intervención socio educativo integrado de formación humana, capacitación e intervención organizacional usado como herramienta para generar cambios en la organización con cinco fases: Co-apreciación situacional, contexto de cambio organizacional, habilidades personales del directivo, investigación-acción: problemas y soluciones y evaluación del proceso

Elabora modelo de consultoría para Mipymes con siete etapas (diagnóstico, focalización del proceso a mejorar, modelado de la situación actual, modelado de la situación propuesta, análisis de propuestas con el equipo de cambio, crear plan de acción y de contingencias y gestión del cambio

Propone modelo de proyecto de gestión del conocimiento basado en factores claves estratégicos, tecnológicos y culturales, con cinco etapas: Análisis de la situación actual, diseño de estrategia, diseño solución, implantación y gestión y evaluación
Proceso, formación y capacitación, sistema de información, entorno, mejora continua, calidad del servicio, satisfacción de las necesidades y expectativas del cliente, cambio, retroalimentación

Cambio, entorno, proceso, mejora, retroalimentación, formación, entrenamiento, capacitación

Entorno, cambio, procesos, sistema de información, mejora continua, calidad del servicio, satisfacción de las exigencias del cliente, capacitación

Calidad del servicio, procesos, satisfacción y expectativas del cliente, cambio, capacitación, mejora continua

Capacitación, aprendizaje, formación, cambio, proceso, calidad y satisfacción del usuario externo e interno

Entorno, procesos, mejora continua, cambio, retroalimentación, satisfacción y expectativas del cliente, sistema automatizado, capacitación, identificación de riesgos

Proceso, retroalimentación, aprendizaje y formación, mejora continua, necesidades y expectativas del cliente, calidad del servicio, cambio, entorno, sistemas de información 


\section{Cont... Cuadro 1.}

Arzola \& Mejias (2010)

11 Flores (2011)

Antúnez

14 \& Franch

(2011)
Propone metodología de consultoría con cinco etapas para la organización de proyectos civiles 4520 C.A. (Inicio, planificación, ejecución, seguimiento y control y cierre

Presenta seis fases para el servicio consultivo (Detección de necesidades, diseño de soluciones, propuesta, oferta de servicio, diagnóstico, plan de acción y ejecución), basado en la teoría de solución de problemas como base para el aprendizaje organizacional

Propone cinco etapas para consultoría: Detección del problema, definición del problema, elaboración de soluciones, implantación de soluciones y evaluación de resultados, ubica dentro de las etapas las características de crítica, juicio y acción

Aplica método inductivo a una propuesta específica para la integración de procesos empresariales y de producción en un laboratorio académico, desarrolla el marco metodológico en consultoría de tecnologías de la información y el estudio de casos

Propone factibilidad de negocio para la creación de una empresa consultora que provea servicios de diseño de programas integrados de responsabilidad social empresarial

Diseña un modelo de diagnóstico que integra las consultorías realizadas con las líneas de investigación trazadas, compuesto por tres etapas: Revisión bibliográfica, análisis y procesamiento de la información y propuesta de solución. Presenta un mapa conceptual de la consultoría gerencial

Diseña una propuesta metodológica para la realizar consultorías en estrategia organizacional con tres etapas: Apertura, desarrollo del proceso consultivo y cierre, que se despliegan en ocho fases y 25 pasos

Propone modelo de aprendizaje organizacional de cinco categorías de análisis habilidades para la dimensión individual y organizacional, con una estrecha relación que determina la capacidad de aprendizaje, la interacción y retroalimentación entre los factores externos e internos, es decisiva para lograr el objetivo
Mejora continua, procesos, calidad del servicio, necesidades y expectativas del cliente, manejo de riesgos, capacitación, cambio

Cambio, entorno, procesos, aprendizaje, formación, calidad del servicio, satisfacción del cliente, marco legal o legislación, mejora continua

Entorno, cambio, procesos, mejora, capacitación, satisfacción del cliente, retroalimentación

Procesos, sistemas de información, entorno, capacitación, mejora continua, cambio, calidad del servicio, satisfacción del cliente

Entorno, proceso, responsabilidad social empresarial, marco legal, calidad del servicio, retroalimentación, satisfacción del cliente, mejora de procesos, capacitación

Proceso, cambio, capacitación, mejora, formación, entrenamiento, aprendizaje, responsabilidad social empresarial, retroalimentación, sistemas de información, calidad del servicio, satisfacción al cliente

Proceso, capacitación, retroalimentación, satisfacción de las necesidades y expectativas del cliente

Proceso, capacitación, formación aprendizaje, retroalimentación, calidad del servicio, entorno, cambio, mejora de procesos, sistemas de información 


\section{Cont... Cuadro 1.}

(Romero, 2012)

Colectivo

18

e autores

(2012)

Centro de Innovación en Tecnolo-

gía para el

Desarrollo

Humano

(2014)

Bahamón

Asesores

Asociados

S.A (2015)
Diseña un conjunto de indicadores financieros y no financieros para medir el comportamiento de los procesos básicos, de apoyo y de crecimiento de la empresa

Propone cuatro etapas para identificar evaluar los problemas y causas existentes en la cultura organizacional de una empresa mixta: preparatoria, formación-acción, intervención-acción, ajustes y fundamentación

Propone un procedimiento para estudios de caso con cinco pasos (Iniciación del trabajo, preparación para la visita, realización de la visita, confección del estudio y difusión del trabajo), incluye herramientas para la recopilación de datos y listas de comprobación

Propone tres etapas para lograr el mejoramiento organizacional (Diagnóstico institucional y del entorno, propuesta de mejoramiento y plan de implementación), además de una metodología de ocho pasos para desarrollar la planeación estratégica, con capacidad de adaptación a otras empresas

Propone un modelo para desarrollar la consultoría en cinco etapas: Concertación del servicio, exploración, planificación, ejecución, informe final, entrega y conformación del expediente
Entorno, proceso, cambio, mejora continua, satisfacción de las necesidades y expectativas del cliente, capacitación, sistema de control, calidad del servicio

Entorno, procesos, cambio, mejora, satisfacción del cliente, capacitación, formación-acción, sistema de comunicación - buzón electrónico, retroalimentación

Entorno, cambio, aprendizaje

Entorno, procesos, mejora continua, marco legal, sistema de información, capacitación, cambio, satisfacción del cliente, calidad del servicio

Enfoque a procesos, retroalimentación, mejora continua, cambio, capacitación, entorno, calidad del servicio y satisfacción de cliente

Fuente: elaboración propia

Del análisis anterior se obtiene que 13 de los autores estudiados establecen un ciclo para el desarrollo de la consultoría, que representa el $62 \%$, por lo que se concluye que para lograr uniformidad en la realización de la consultoría se deben establecer pasos secuenciales que guíen la manera de hacer. Asimismo, se identifican las variables utilizadas para caracterizar el proceso consultivo desde la mirada de cada autor.

\section{Variables más representativas utilizadas en el proceso de consultoría}

Se desarrolla un proceso de análisis y síntesis a los mencionados instrumentos metodológicos para conocer las variables más representativas. Derivadas del análisis, se observa la presencia de catorce variables manejadas indistintamente por los autores. 


\section{Cuadro 2.}

\section{Variables presentes en los instrumentos metodológicos.}

\begin{tabular}{|c|c|c|}
\hline Código & Variable & Forma de expresión \\
\hline 1 & Enfoque a proceso & $P$ \\
\hline 2 & Retroalimentación & $\mathrm{R}$ \\
\hline 3 & Mejora/mejora continua & M \\
\hline 4 & Cambio organizacional & C \\
\hline 5 & Capacitación/formación & $\mathrm{Cp}$ \\
\hline 6 & Aprendizaje & A \\
\hline 7 & Entrenamiento & $\mathrm{E}$ \\
\hline 8 & Entorno & En \\
\hline 9 & Calidad del servicio & Q \\
\hline 10 & Satisfacción del cliente & Scl \\
\hline 11 & Sistema de Información / control / comunicación & $\mathrm{Si}$ \\
\hline 12 & Identificación de riesgos & Ir \\
\hline 13 & Responsabilidad social empresarial & Rse \\
\hline 14 & Marco legal & $\mathrm{Ml}$ \\
\hline
\end{tabular}

Fuente: Elaboración propia.

Los instrumentos metodológicos consultados son evaluados de acuerdo a las variables definidas. Los datos se procesan a partir de la construcción de una matriz binaria, con la presencia (1) o ausencia (0) de las variables en cada instrumento, el análisis realizado se expone en la tabla 1. 


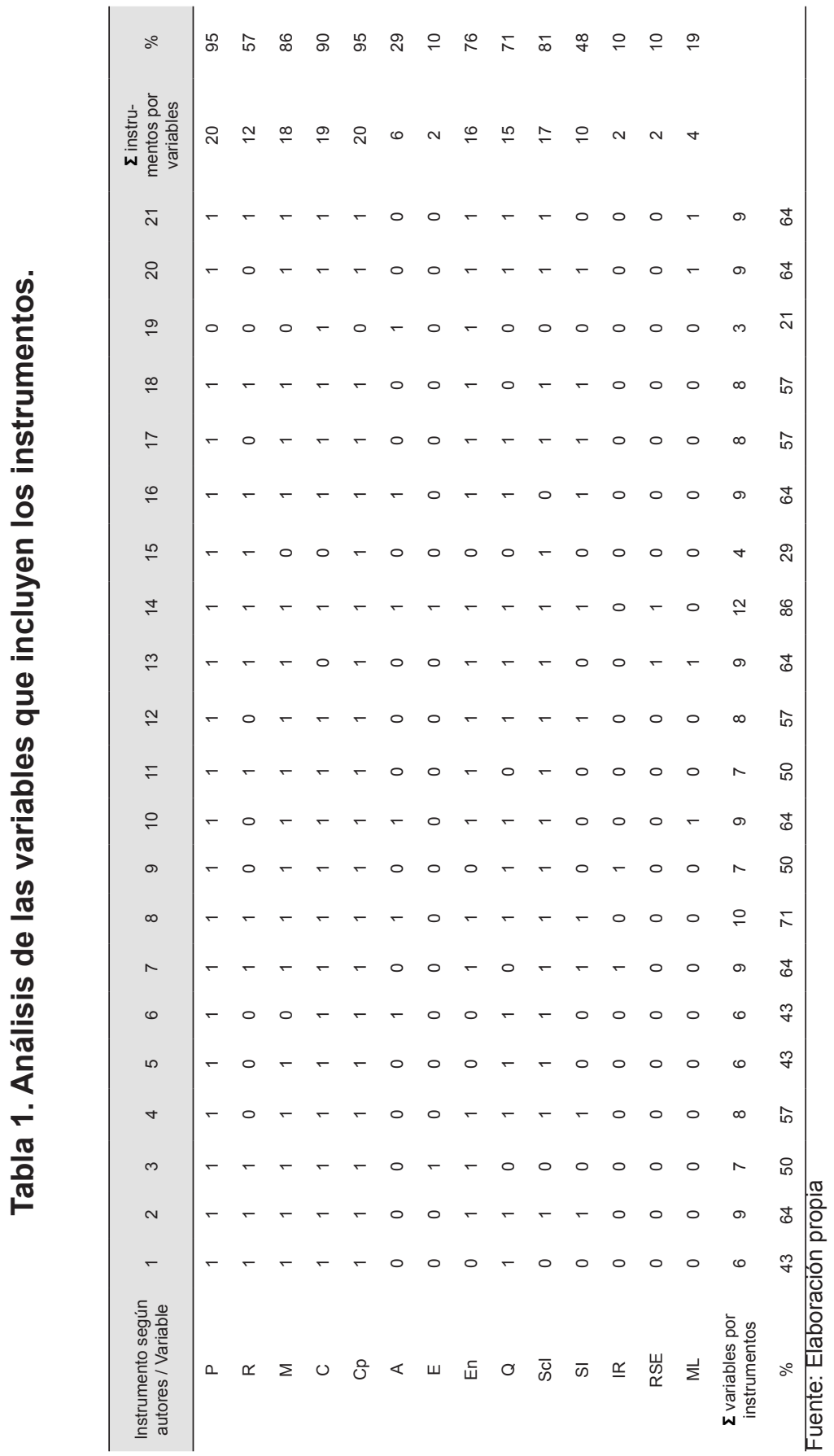


Esta información se procesa mediante el IBM SPSS Statistics versión 21 , se utiliza la metodología clúster y el análisis de clasificación por conglomerados jerárquicos por casos, según el método Ward e intervalo de distancia euclídea al cuadrado. Como resultado se obtienen el dendograma mostrado la figura 1.

\section{Figura 1. \\ Dendograma del análisis clúster para los 21 instrumentos estudiados.}
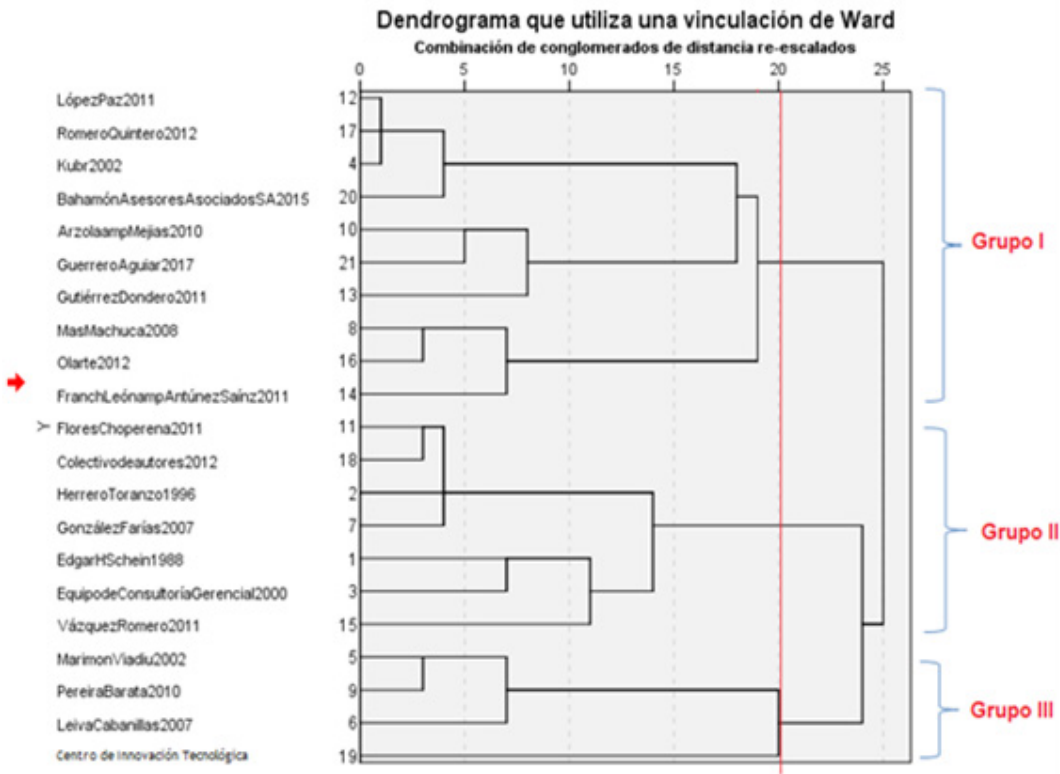

Fuente: Elaboración propia
Como puede observarse a una distancia de aglomeración igual a veinte (20), se obtienen tres grupos:

El primero, está conformado por diez instrumentos (48 \% ): 1) López (2011), con una propuesta para la integración de procesos empresariales y procesos de producción en un laboratorio académico; 2) Romero (2012), con una metodología para desarrollar la consultoría económica; 3) Kubr (2002), que detalla su modelo de consultoría; 4) Bahamón Asesores Asociados S.A
(2015), que propone una metodología para desarrollar la consultoría como proceso de intervención sistémica; 5) Arzola and Mejias (2010), metodología para la realización del servicio consultivo basado en la teoría de solución de problemas; 6) Guerrero (2017) modelo de consultoría organizacional; 7) Gutiérrez (2011), con un plan de negocios para servicios de consultoría de responsabilidad social empresarial; 8) Mas (2008), propone un modelo de sistema basado en los factores clave para 
la gestión del conocimiento; 9) Olarte (2012), con un modelo de aprendizaje organizacional y; 10) Antúnez \& Franch (2011), con una propuesta metodológica para desarrollar el diagnóstico.

En este grupo se concentran los instrumentos que abordan mayor cantidad de variables (doce), de ellas, cinco comunes para todos los instrumentos, es el más representativo.

El segundo grupo está compuesto por siete instrumentos (33 \%): 1) Flores (2011), con metodología para el desarrollo de estudios de caso; 2) Colectivo de autores (2012), metodología para la mejora de la cultura organizacional;3) Herrero (1996), con un modelo de intervención socio educativo; 4) González (2007), con un modelo de consultoría para las MyPimes; 5) Schein (1988), modelo de consultoría de procesos; 6) González (2000), modelo de consultoría organizacional-gerencial; y 7) Vázquez (2011), con una metodología para la realización de consultorías en estrategia organizacional. Muestra tres variables comunes en todos los instrumentos y tres ausentes.

El tercer grupo lo componen cuatro instrumentos (19\%): 1) Marimon (2002), trabaja la consultoría desde los distintos modelos de gestión de calidad; 2) Pereira (2010), metodología para el desarrollo de los servicios de consultoría en gerencia de proyecto; 3) Leiva (2007) con una propuesta de modelo de intervención socio educativa y; 3) Centro de Innovación en Tecnología para el Desarrollo Humano (2014), con una propuesta para la consultoría estratégica. En este grupo se aprecia la ausencia de cinco variables y una sola variable presente en todos los instrumentos que es el cambio organizacional.

En la tabla 2 se resumen los conglomerados por grupos de autores, donde se destacan las variables más representativas.

Tabla 2.

Análisis del conglomerado por grupos de instrumentos.

\begin{tabular}{|c|c|c|c|c|c|}
\hline $\begin{array}{l}\text { O } \\
\text { ํํㄹ } \\
\text { i }\end{array}$ & 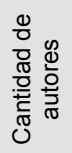 & $\begin{array}{c}\text { Autores que abordan mayor } \\
\text { cantidad de variables }\end{array}$ & $\%$ & $\begin{array}{l}\text { Variables más } \\
\text { representativas }\end{array}$ & $\frac{\frac{\pi}{0}}{2}$ \\
\hline 1 & 10 & $\begin{array}{l}\text { (Antúnez \& Franch 2011), } \\
\text { (Mas, 2008), (Arzola \& Mejias, } \\
2010),(\text { Gutiérrez, 2011), (Olar- } \\
\text { te, 2012), (Bahamón Asesores } \\
\text { Asociados S.A, 2015) y Guerre- } \\
\text { ro, } 2017\end{array}$ & 48 & $\begin{array}{l}\text { Enfoque al proceso, mejora, cambio organi- } \\
\text { zacional, capacitación, entorno, calidad del } \\
\text { servicio, Satisfacción del cliente }\end{array}$ & 6,5 \\
\hline 2 & 7 & $\begin{array}{l}\text { (Herrero, 1996), (González, } \\
\text { 2007), (Colectivo de autores, } \\
\text { 2012) }\end{array}$ & 33 & $\begin{array}{l}\text { Enfoque al proceso, retroalimentación y } \\
\text { capacitación }\end{array}$ & 3,57 \\
\hline 3 & 4 & $\begin{array}{l}\text { (Pereira, 2010), (Marimon, } \\
\text { 2002), (Leiva, 2007) }\end{array}$ & 19 & Cambio organizacional & 1,57 \\
\hline
\end{tabular}

Fuente: Elaboración propia 
Asimismo, del análisis del conglomerado por grupos de variables (figura 2) se aprecia, a una distancia igual a ocho, la formación de dos grupos: el primero, con nueve variables $(64 \%)$, donde se manifiesta que las variables con mayor presencia son enfoque a proceso, capacitación, cambio organizacional, mejora continua, satisfacción de cliente, entorno y calidad del servicio; y, el segundo, formado por cinco variables ( $36 \%)$.

Figura 2. Dendograma del análisis clúster por variables

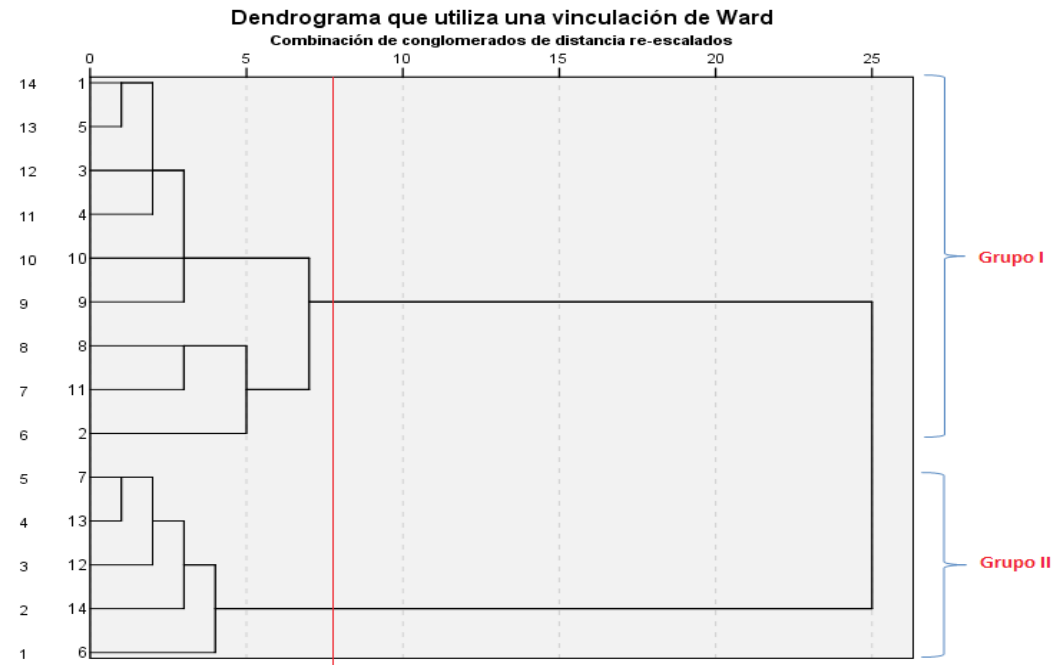

Fuente: Elaboración propia

Para una mayor comprensión se la tabla 3. consolidan las variables por grupos en

Tabla 3.

Análisis del conglomerado por grupos de variables

\begin{tabular}{|c|c|c|c|c|}
\hline $\begin{array}{l}\text { : } \\
\text { 을 } \\
\text { 니 }\end{array}$ & 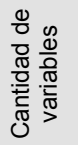 & Variables más representativas & $\begin{array}{l}\% \text { de variables } \\
\text { que utilizan }\end{array}$ & $\frac{\frac{\pi}{\sigma}}{\sum}$ \\
\hline 1 & 9 & $\begin{array}{l}\text { Enfoque al proceso, mejora, cambio organizacional, capaci- } \\
\text { tación, entorno, calidad del servicio, satisfacción del cliente }\end{array}$ & $64 \%$ & 16,33 \\
\hline 2 & 5 & Aprendizaje y marco legal & $36 \%$ & 3 \\
\hline
\end{tabular}

Fuente: Elaboración propia 
Como puede apreciarse, tanto en el análisis de conglomerados por instrumentos metodológicos, como por variables, coinciden las variables más representativas, por lo que se concluye que existe consenso entre los autores acerca de las variables a considerar para desarrollar el proceso de consultoría.

\section{Trabajo grupal y análisis crítico con los consultores}

En sesión de trabajo grupal con el equipo de consultores con experiencia en esta actividad, se realiza un análisis crítico de los instrumentos metodológicos, se exponen los resultados alcanzados hasta el momento (fases o etapas e invariantes). La información es analizada mediante tormenta de ideas con el objetivo de entender la lógica y secuencia de las diversas etapas propuestas, contrastarlas con las etapas utilizadas en la práctica del consultor y arribar a consenso, asimismo, comprender el proceso tomando como base las variables utilizadas para definirlo.

En este sentido, se entrega una encuesta con todas las etapas y variables propuestas en los instrumentos metodológicos, para que cada miembro del equipo marque las que a su criterio debe contener el modelo. Resultado que se presenta al grupo para reducir el listado, ponderar las propuestas y llevarlas a votación. En consecuencia, se procede a determinar otras exigencias que debe tener el modelo para desarrollar el proceso de consultoría en las entidades. El equipo de trabajo determina lo siguiente:

1. De las etapas encontradas en la literatura, el modelo debe contener las siguientes: concertación del servicio, exploración, planificación, ejecución e informe gerencial, entrega y conformación del expediente.

2. Debe contemplar las siete variables más representativas: enfoque al proceso, mejora, cambio organizacional, capacitación, entorno, calidad del servicio, satisfacción del cliente.

3. Reflejar la consultoría como proceso, con sus entradas, transformación y salidas.

4. Sustentarse en el ciclo de mejora continua de Deming (PHVA).

5. Contemplar los cambios del entorno (variación de las condiciones y variables que le dieron origen).

Elementos que se enriquecen y fertilizan con las experiencias de los consultores hasta arribar a la concepción del modelo.

\section{Propuesta de modelo de consultoría}

Como resultado del análisis de los conceptos, las fases o etapas tradicionalmente utilizadas para desarrollar la consultoría, la articulación de las variables con mayor frecuencia de aparición y la experiencia teóricopráctica acumulada por el equipo consultor se propone modelo que se muestra en la figura 3 , 
Figura 3.

Modelo de consultoría organizacional

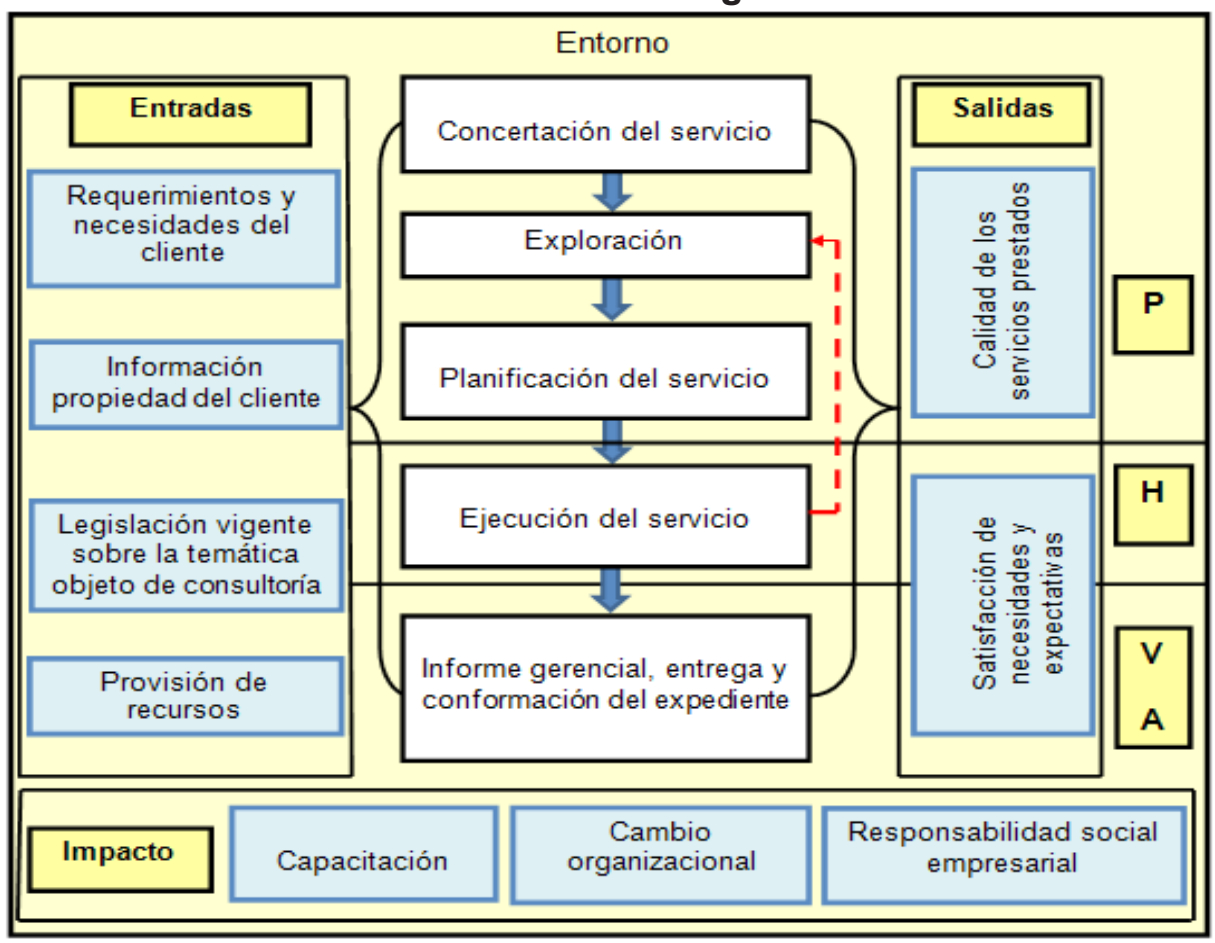

Fuente: Elaboración propia.

El objetivo del modelo consiste en ofrecer una guía a los consultores que les permita la presentación de soluciones viables a los problemas prácticos y complejos que presentan las organizaciones.

\subsection{Características del modelo:}

- Formativo: Se instruye a las personas a partir del aprendizaje mediante la formación - acción, se aplican acciones de entrenamiento, según las necesidades que se presenten, para proveer de habilidades a los equipos de trabajo.

- Flexible: El modelo brinda la posibilidad de ser adaptado a la realidad de la empresa objeto de consultoría, mediante el empleo de las herramientas más acertadas.

- Promotor de la mejora continua: Se materializa en la solución de problemas centrados en el mejoramiento continuo a partir de la retroalimentación al sistema que permite realizar los ajustes necesarios al proceso.

- Pertinencia: Dado por la posibilidad 
de ser aplicado totalmente según los intereses de la organización

\subsection{Principios que respaldan el modelo:}

1. Participativo: Durante todo el proceso consultivo se trabaja en equipo, se genera cohesión entre sus miembros, se ven involucrados tanto el consultor como el cliente, este último participa en la aplicación de técnicas, herramientas, propone soluciones y evalúa las propuestas realizadas por el consultor.

2. Creatividad: En la relación cliente consultor se establece un ambiente de colaboración que favorece la propuesta de soluciones novedosas a partir de informaciones ya conocidas, el hallazgo de problemas aún no detectados y selección de la mejor alternativa de solución.

3. Consistencia lógica: Se evidencia en la disposición, conformación secuencial de los elementos que integran el modelo y su interrelación, es coherente con la lógica de ejecución del proceso consultivo.

\subsection{Premisa en la que se sustenta el modelo}

1. Tener autorización de la máxima dirección para ejecutar el proceso de consultoría.

El modelo propuesto tiene un enfoque de procesos con base en el ciclo de mejora continua de Deming (PHVA), al mismo tiempo, está sujeto a la observancia constante del entorno para tomar medidas de carácter proactivo.

Considera como variables de entrada los requerimientos y necesidades del cliente; la información propiedad del cliente; la legislación vigente y la provisión de recursos. La transformación viene dada por los pasos para desarrollar el proceso de consultoría y entre las variables de salidas se encuentran la satisfacción de necesidades y expectativas del cliente y la calidad de los servicios prestados. El proceso de transformación contempla cinco fases.

\subsection{Fases del modelo propuesto}

\section{Fase I. Concertación del servicio.}

En esta fase se establecen las primeras relaciones entre el consultor y el cliente, se conocen sus necesidades, en caso de tratarse de un nuevo cliente, se conforma la ficha a fin de que quede registrado en la base de datos de clientes de la empresa consultora y se ponen de acuerdo las partes en cuanto al alcance, condiciones, recursos, personal y la documentación necesaria para comenzar el servicio.

\section{Fase II. Exploración.}

En esta fase se realiza un diagnóstico a partir de la información suministrada por el cliente, con el objetivo de conocer el funcionamiento de la entidad y el estado de sus procesos, para constatar la existencia de los problemas enunciados previamente por éste, resultados que deben quedar reflejados como evidencia del trabajo realizado.

\section{Fase III. Planificación del servicio.}

En esta fase se planifican las 
acciones de capacitación que necesita el cliente sobre el tema objeto de consultoría, de las herramientas y técnicas que se van a utilizar durante el servicio, así como de los recursos que demandan estas acciones.

\section{Fase IV. Ejecución del servicio.}

Durante esta fase se elaboran los papeles de trabajo para dar solución al problema, que son la evidencia de los muestreos realizados, de la aplicación de herramientas y técnicas, del empleo de normas y resoluciones vigentes tanto internas como externas y de la solución elegida por el cliente para resolver el problema identificado.

Posteriormente se elaboran los documentos de cierre del servicio y el informe preliminar con los resultados del trabajo realizado, el cual se discute con las partes para lograr su conformidad.

\section{Fase V. Informe gerencial, entrega y conformación del expediente.}

En esta fase se conforma el informe gerencial, que se entrega de conjunto con la documentación del servicio realizado al cliente para su firma, se realiza la entrega formal de la documentación propiedad del cliente, además se tramita la facturación, el cobro del servicio, la opinión del cliente sobre el servicio recibido. De igual forma, se conforma el expediente de consultoría que es sometido a un proceso de revisión de todos sus documentos, luego se procede al archivo del expediente y finalmente, se salva de la información.

El modelo concibe la retroalimentación al sistema, de la fase de ejecución a la de exploración, donde, a partir de las experiencias prácticas adquiridas (aciertos y desaciertos), durante la ejecución de la consultoría se aplican mejoras e incorpora valor a los nuevos servicios.

Combina la gestión de las variables capacitación, cambio organizacional y responsabilidad para impactar positivamente en la entidad cliente de la forma siguiente:

- Capacitación, con acciones formativas tanto para directivos, como para trabajadores, que les permitan apropiarse de la teoría, marco legal, técnicas, herramientas e involucrarse en el proceso para aprender a identificar y resolver sus propios problemas.

- Cambio organizacional, con el arribo al estado deseado, a mediano o largo plazo, luego de haber aplicado las acciones recomendadas por el consultor, es el elemento concluyente que resulta del proceso consultivo, razón por la cual se da inicio a la consultoría.

- Responsabilidad social empresarial, expresada en la contribución al comportamiento ético responsable ante la sociedad a partir de la aplicación de estrategias y buenas prácticas de gestión empresarial para contribuir a la mejora económica, ambiental y social.

La propuesta de modelo se encuentra articulada al marco legal, lo que permite realizar una mirada secuencial de los servicios demandados en busca de la satisfacción del cliente, al brindar servicios de mayor calidad. Contempla las siete variables con mayor frecuencia de aparición. Sin embargo, a pesar de que la responsabilidad social empresarial alcanza solo un $10 \%$ de 
representatividad, se considera una variable importante, desde la óptica de la repercusión que tiene la consultoría en el comportamiento ético y responsable de la organización cliente ante la sociedad Medina (2016), Fraga et al (2016), Jaquinet (2016).

El modelo resultado de esta investigación aprovecha y sistematiza las buenas prácticas existentes en el equipo consultor.

\section{Conclusiones}

Se realiza el estudio de 21 instrumentos metodológicos que sirven de basamento para el modelo de consultoría propuesto, que aprovecha las buenas prácticas adquiridas, las integra en una herramienta de trabajo que contribuye a conservar y transmitir el conocimiento a los diferentes actores de esta área del conocimiento.

$\mathrm{Se}$ propone un modelo caracterizado por la integración de herramientas, que logra uniformidad al sistematizar la forma de desarrollar los servicios de consultoría, que se articula con el merco legal, orientado al cliente, compuesto por cinco fases: concertación del servicio, exploración, planificación, ejecución e informe gerencial, entrega y conformación del expediente.

\section{Referencias bibliográficas}

Antúnez Saínz, Vivian \& Franch León, Katia (2011), La consultoría como forma de actividad científica en el CETED: una propuesta para su perfeccionamiento, Universidad de la Habana, Cuba, Disponible: http:// www.redciencia.cu/geprop/index. html [Consulta: 2018, Abril 21].

Arzola Hamilton, Minerva \& Mejias
López, Agustin (2010), Morfologia del aprendizaje y la gestion del conocimiento del sector servicios de consultoría, Revista Ingeniería Industrial, Año 9, N 1, Cuba, ISPJAE, pp 37-51.

Asamblea General Naciones Unidas (2015), Resolución 70/1, Transformar nuestro mundo: la agenda 2030 para el Desarrollo Sostenible, Nueva York. Estados Unidos.

Bahamón Asesores Asociados S.A. (2015), Propuesta de consultoría para redefinir los lineamientos estratégicos de la Comisión Latinoamericana de Aviación Civil, Bogota, Colombia.

Centro de Innovación en Tecnología para el Desarrollo Humano (2014). Metodología para el desarrollo de estudios de caso, Universidad Politécnica de Madrid. España.

Flores Choperena, Oscar Everardo (2011), La consultoría como proceso de intervención sistémica. Tesis de maestría en Ingeniería, Universidad Nacional Autónoma de Mexico, Mexico.

Fraga Domínguez, Leovaldo et al (2016), De los escándalos financieros a la Responsabilidad Social Empresarial en las Casas Consultoras y de Auditoría. Una mirada desde la realidad cubana, Memorias CD-R II Congreso Internacional de Gestión Económica y Desarrollo, ANEC, ISBN: 978-95907-2123-6, Habana, Cuba.

González Farías, José Porfilio (2007), Modelo de Consultoría para las Micro, Pequeñas y Medianas Empresas de Guanajuato, México, (Tesis de doctorado), ISPJAE, Habana, Cuba.

González Méndez, Laredo (2000), Consultoría organizacional-gerencial y el consultor. En Consultoría gerencial y cambio organizacional. Una 
contribución al perfeccionamiento empresarial. Tomo I y II, pp 93-100, Universidad de la Habana. Cuba.

Guerrero Aguiar, Margarita et al (2014), Consultoría de gestión y prevención de riesgos, Memorias CD-R Conferencia Internacional de Ciencias Empresariales, Universidad Central de las Villas Marta Abreus, Santa Clara, Cuba, ISBN: 978-959-250-995-5.

Guerrero Aguiar, Margarita (2017), Modelo de consultoría para gestionar los servicios que brinda Audita S.A. al sector empresarial, Paper presented at the Segundo Congreso Internacional Virtual sobre Los Modelos Latinoamericanos de Desarrollo, Universidad de Málaga, España. Eumed.net. ISBN-13: 978-84-1687445-3, pp 190-198.

Guerrero Aguiar, Margarita et al (2018), Diseño de un procedimiento general de consultoría organizacional, Revista Universidad y Sociedad, Vol. 10, No.5, Cuba, Universidad de Cienfuegos, pp 21-33. Disponible: http:// rus.ucf.edu.cu/index.php/rus [Consulta: 2018, Octubre 31].

Gutiérrez Dondero, Julieta Isabel (2011), Primera etapa plan de negocios en Chile para servicios de consultoría de diseño de programas integrados de Responsabilidad Social Empresarial, (Tesis de maestría), Universidad de Chile, Chile.

Herrero Toranzo, Emiliano (1996), Aportaciones de las ciencias humanas a la consultoría de procesos integrada para impulsar el desarrollo y planteamiento estratégico de las organizaciones, (Tesis de doctorado), Universidad Complutense de Madrid, España.

Jaquinet Espinosa, Rebeca Milenys (2016), Contribución al control de gestión en las instituciones de educación superior a través de la comu- nicación organizacional, (Tesis de doctorado), Universidad de Matanzas, Cuba.

Kubr, Milán (2002), Management Consulting: A guide to the profession (Fourth ed), Geneva: International Labour Office, pp. 948.

Leiva Cabanillas, Jorge (2007), Fundamentación y diseño de un modelo de intervención socio educativa desde una perspectiva constructivista, para su aplicación en organizaciones productivas o de servicios. Estudio de su aplicación y observación de su impacto en una empresa, (Tesis de doctorado), Universitad Ramon Lull, Barcelona, España.

López Paz, Carlos Ramón (2011), Metodología para la Sistematización de los Servicios de Consultoría TI. (Tesis de doctorado), Universidad de Alicante, España.

Marimon Viadiu, Frederic (2002), La consultoría especializada en ISO 9000 en Cataluña: calidad del servicio y beneficios, (Tesis de doctorado), Universidad de Girona, España.

Mas Machuca, Marta (2008), Modelo de sistema basado en los factores clave para el diseño e implementación de proyectos de Gestión del Conocimiento. Aplicación al sector de la consultoría, (Tesis de doctorado), Universidad Autónoma de Cataluña, España.

Medina Nogueira, Daylin (2016), Instrumento metodológico para gestionar el conocimiento mediante el observatorio científico, (Tesis de doctorado), Universidad de Matanzas, Cuba.

Olarte Moyano, Juan Carlos (2012), Aprendizaje organizacional y proceso de consultoría, Voces y Silencios: Revista Latinoamericana de Educación, Vol. 3, No. 1, Universidad de los Andes, Colombia, pp 7086. 
Pereira Barata, David Hernani (2010). Diseño de servicio de consultoría en gerencia de proyecto para la Empresa de Proyectos Civiles 4520 C. A. (Tesis de Especialista en Gerencia de Proyectos), Universidad Católica Andrés Bello, Caracas, Venezuela

Colectivo de autores (2012), La consultoría como instrumento para la integración y la mejora de la cultura organizacional en una empresa (Proyecto conexión), Revista Cofin Habana, N. ${ }^{\circ} 4$, Cuba, Editorial UH, pp $8-16$.

Romero Quintero, Daylet Iliana (2012), Aplicación de Metodología de la Consultoría Económica del MINTUR para evaluar la gestión empresarial del
Complejo Hotel E La Unión - Palacio Azul, (Tesis de Grado), Universidad de Cienfuegos, Cuba.

Schein, Edgar H. (1988), Consultoría de Procesos. Recomendaciones para Gerentes y Consultores (Segunda ed. Vol. 2), Argentina: Addison-Wesley, pp 169.

Vázquez Romero, Javier Eduardo (2011), La consultoría en estrategia organizacional en el CETED: una experiencia en la Empresa de la Goma, POLIGOM, (Tesis de maestría), Universidad de la Habana, Cuba.

- Esta obra está bajo una licencia de Creative Commons Reconocimiento-NoComercialCompartirlgual 3.0 Unported.

http://creativecommons.org/licenses/by-nc-sa/3.0/deed.es_ES 